\title{
Acciones didácticas para el logro de buenas prácticas inclusivas
}

\section{Didactic Actions for the Achievement of Inclusive Good Practices}

\author{
Raúl González Peña* \\ Universidad Central "Marta Abreu de Las Villas", Villa Clara, Cuba. \\ ORCID: https://orcid.org/0000-0003-4428-9398 \\ María Antonia Monjas Arteaga \\ Universidad Central "Marta Abreu de Las Villas", Villa Clara, Cuba. \\ ORCID: https://orcid.org/0000-0001-5972-2962 \\ Lina Lutgarda Alba Benítez \\ Universidad Central "Marta Abreu de Las Villas", Villa Clara, Cuba. \\ ORCID: https://orcid.org/0000-0001-7232-7027

\section{Dunia Yudit Santos Fernández} \\ Universidad Central "Marta Abreu de Las Villas", Villa Clara, Cuba. \\ ORCID: https://orcid.org/0000-0001-7412-6931
}

Received 03-12-20 Revised 05-15-20 Accepted 09-20-20 On line 09-30-20

*Correspondence

Email: raulgp@uclv.cu
Cite as:

González, R., Monjas, M. A., Alba, L. L., \& Santos, D. Y. (2020). Acciones Didácticas para el Logro de Buenas Prácticas Inclusivas. Propósitos y Representaciones, 8 (SPE3), e727. Doi: http://dx.doi.org/10.20511/pyr2020.v8nSPE3.727 


\section{Resumen}

La educación inclusiva se ha convertido en una alternativa cierta y eficaz para atender a las personas con necesidades educativas especiales (NEE) que se escolarizan desde la educación regular, enfocando su actuación, hacia la transformación radical de las instituciones educativas para eliminar las barreras que atentan contra el acceso, la participación, el aprendizaje y el desarrollo integral de todo el alumnado. Precisamente, el enorme desafío que tienen ante sí los sistemas educativos para lograr comunidades inclusivas les exige la instrumentación articulada de políticas coherentes, de una extendida cultura de la diversidad y de buenas prácticas inclusivas. Sin embargo, son las buenas prácticas inclusivas materializadas en estrategias pedagógicas, las que en última instancia deciden el éxito o el fracaso de este complejo proceso. Al respecto se ofrece la visión de un colectivo de investigadores que han profundizado en esta problemática, quienes comparten una alternativa conformada por un sistema de acciones didácticasmetodológicas dirigidas a elevar la calidad del proceso de enseñanza-aprendizaje que se desarrolla en condiciones de inclusión educativa.

Palabras clave: Inclusión educativa, NEE, Prácticas inclusivas.

\section{Summary}

Inclusive education has become a true and effective alternative to serve people with special educational needs (SEN) who are enrolled from regular education, focusing their actions, towards the radical transformation of educational institutions to eliminate the barriers that threaten against access, participation, learning and integral development of all students. Precisely, the enormous challenge facing educational systems to achieve inclusive communities requires them to articulate coherent policies, an extended culture of diversity and good inclusive practices. However, it is good inclusive practices embodied in pedagogical strategies, which ultimately decide the success or failure of this complex process. In this regard, we offer the vision of a group of researchers who have deepened in this problem, who share an alternative formed by a system of didacticmethodological actions aimed at raising the quality of the teaching-learning process that takes place in conditions of educational inclusion.

Keywords: educational inclusion, SEN, inclusive practices.

\section{Introducción}

El proceso de atención psicopedagógica al alumnado con necesidades educativas especiales en contextos de inclusión educativa ha suscitado en los últimos años, la mirada crítica de los docentes y de los profesionales encargados de garantizar la educación de este grupo social vulnerable. Sin embargo, en la actualidad el debate ha tomado mayor realce debido a la ampliación del campo de la educación inclusiva hasta sectores sociales donde siempre existió una exclusión manifiesta, tanto en el ámbito de la escuela, como en el propio contexto social.

Autores como Castel (1995) advierten la relación dialéctica entre inclusión, exclusión y un importante constructo que ocupa una posición intermedia entre ellos, "la vulnerabilidad". Para este autor la exclusión es vista como la condición provisoria y no definitiva en la que se hallan los sujetos excluidos, ya sea por razones culturales, sociales, psicológicas o por alguna discapacidad física; y sobre todo, es admitida no como una faceta determinante en la vida de los seres humanos o como un lugar encasillado desde donde no se puede salir, sino que solo expresa un retardo en el curso del desarrollo que puede ser resuelto, precisamente, por el cambio paradigmático que hoy puede ofrecer la educación inclusiva.

Esta es la razón fundamental por la que la Educación Especial contemporánea ha cambiado su enfoque de actuación, desde una misión centrada en brindarle respuesta a las necesidades educativas especiales, hacia la identificación y eliminación de las barreras que 
afectan la presencia, la participación y el aprendizaje del alumnado con dichas necesidades. (Booth \& Ainscow, 2002).

Ahora bien, para comprender a cabalidad el alcance, la magnitud y la complejidad que encierra el proceso de inclusión socioeducativa del alumnado con necesidades educativas especiales (NEE) se hace necesario precisar de forma analítica la esencia de este concepto y los elementos que le distinguen. El término inclusión es un vocablo que proviene del latín inclusio, -ōnis, y se define como la conexión o amistad de alguien con otra persona (Diccionario de la lengua española, 2014).

A su vez la UNESCO (2008) sobre este tema considera que,

(...) puede ser concebida como un proceso que permite abordar y responder a la diversidad de las necesidades de todos los educandos a través de una mayor participación en el aprendizaje, las actividades culturales y comunitarias y reducir la exclusión dentro y fuera del sistema educativo. Lo anterior implica cambios y modificaciones de contenidos, enfoques, estructuras y estrategias basados en una visión común que abarca a todos los niños en edad escolar y la convicción de que es responsabilidad del sistema educativo regular para educar a todos los niños y niñas. El objetivo de la inclusión es brindar respuestas apropiadas al amplio espectro de necesidades de aprendizaje tanto en entornos formales como no formales de la educación (p. 8).

Por su parte son muchos los autores que reconocen a la educación inclusiva como un tema de derecho de todo el alumnado a recibir una respuesta educativa de calidad. Al respecto Blanco (2006) plantea lo siguiente:

(...) aspira hacer efectivo para todas las personas el derecho a una educación de calidad, que es la base de una sociedad más justa e igualitaria. La educación es un bien común específicamente humano que surge de la necesidad de desarrollarse como tal, por ello todas las personas sin excepción tienen derecho a ella (p.7).

En esta misma dirección se plantea que la educación inclusiva "es un derecho positivo que, por ello, obliga a las autoridades a crear las condiciones para su disfrute efectivo, removiendo en su caso, las circunstancias u obstáculos que impidan su ejercicio, pues de lo contrario estaríamos ante situaciones de discriminación" (Echeita y Ainscow, 2011, p.31).

Por otra parte, Booth, Ainscow, Black-Hawkins, Vaughan \& Shaw (2002) declaran que la educación inclusiva:

Se entiende como un proceso de desarrollo que no tiene fin, ya que siempre pueden surgir nuevas barreras que limiten el aprendizaje y la participación, o que excluyan y discriminen de diferentes maneras a los estudiantes. No cabe duda de que la respuesta a la diversidad del alumnado es un proceso que no solo favorece el desarrollo de éste, sino también el de los docentes, las familias y los centros educativos mismos (p.7).

Sobre la misma idea de ubicar la esencia de la inclusión educativa dentro de un complejo proceso, versan los criterios de Ramírez (2010), quien señala que se trata de "un conjunto de procesos orientados a aumentar la participación de los estudiantes y las comunidades de las escuelas" (p.158).

Consecuentemente autores como Escribano y Martínez (2013) defienden que:

(...) la inclusión no es un nuevo enfoque. Es más bien un reenfoque, una reorientación de una dirección ya emprendida, un corregir los errores atribuidos a la integración escolar, que subraya la igualdad por encima de la diferencia, que 
no se circunscribe solo al ámbito de la educación, es una idea transversal que está presente en los ámbitos de la vida social, familiar, laboral, etc. (p.17).

Desde este punto de vista la inclusión trasciende el contexto escolar y abarca espacios como la sociedad, toda vez que los centros educativos se comprometan a realizar un análisis crítico sobre lo que se puede hacer para mejorar el aprendizaje y la participación de todos en la escuela y en su entorno (Granada, Pomés \& Sanhueza 2013).

Para una mejor comprensión de la definición de inclusión educativa y el desarrollo de esta en cualquier contexto es necesario precisar cuatro elementos indispensables: la inclusión es un proceso; la inclusión busca la presencia, la participación y el éxito de todos los estudiantes; la inclusión precisa la identificación y la eliminación de barreras; y por último, la inclusión pone particular énfasis en aquellos grupos de alumnos que podrían estar en riesgo de marginalización, exclusión, o fracaso escolar (Ainscow, 2004).

A su vez resultan de gran valor para comprender el alcance del concepto que se analiza los criterios de Prietoni (2016) quien destaca que algunos autores añaden al término de inclusión, a modo de adjetivo, la palabra compuesta socio-educativa, al considerar que este:

(...) hace imprescindible la construcción de la igualdad de oportunidades para el acceso, la permanencia, el aprendizaje y el egreso de los niños, niñas, jóvenes y adultos, a través de las distintas instancias educativas que posibilitan una inclusión social profunda. Esta inclusión socio- educativa se sostiene en los valores de solidaridad y emancipación: solidaridad para dar lugar y atender las situaciones de aquellos sujetos con derechos vulnerados; emancipación como perspectiva de trabajo cuyo horizonte es la construcción de sujetos autónomos y responsables. La perspectiva de inclusión socio-educativa recupera la centralidad de una educación para todos problematizando los viejos formatos anclados en propuestas pedagógicas homogeneizadoras (p. 6).

Estas consideraciones permiten concretar que la inclusión socioeducativa es el proceso dinámico y multifactorial dirigido a garantizar y promover el acceso, la permanencia, el aprendizaje, el desarrollo y el éxito de todo el alumnado bajo presupuestos filosóficos, políticos, culturales y didácticos que conduzcan a fundar un nuevo tipo de institución educativa; institución esta, que sea capaz de promover de forma creativa la participación democrática del alumnado y de los docentes; donde se trabaje bajo un enfoque científico , sistémico e intersectorial para la eliminación de las múltiples barreras que conducen a la segregación; y donde se promuevan de forma coherente y planificada relaciones interpersonales resilientes y desarrolladoras, que contribuyan a que todo el alumnado se forme en un ambiente, cultural y pedagógico inclusivo.

Ahora bien, asumir la inclusión socioeducativa como un proceso dinámico, complejo y multifactorial que pretende transformar radicalmente el tipo de institución educativa que se ha forjado a lo largo de la historia sobre la base, de las relaciones sociales y pedagógicas que en ella se establecen, implicará la reasignación de roles particulares para las dimensiones de este proceso (políticas, culturas y prácticas inclusivas).

Adviértase, que será indiscutible que la articulación sistémica entre estas dimensiones constituirá la condición necesaria y suficiente para que el complejo proceso de inclusión educativa cumpla con su encargo de elevar la calidad de la educación a un nivel en que todo el alumnado acceda, aprenda, egrese y se desarrolle en las instituciones educacionales. Sin embargo, por lo general para los docentes, el problema clave estriba en la preparación que necesitan para instrumentar prácticas inclusivas desde los espacios áulicos (González, 2019). Esto significa que el eslabón de base del proceso de inclusión educativa lo constituyen las buenas prácticas que logren materializarse desde los contextos particulares donde estas se precisen. 
En cuanto al concepto de buenas prácticas, comúnmente se definen estas como las formas óptimas de ejecutar un proceso (inclusión educativa) que pueden servir de modelo para otras organizaciones. Adviértase que este concepto por su extensión se refiere a la calidad integral de cualquier tipo de intervención, no obstante, es posible y pertinente su aplicación al campo de la inclusión educativa, pues él comprende la gestión y los procedimientos que se requieren para la instrumentación de acciones didácticas que promuevan el aprendizaje y la participación de todo el alumnado y que por su naturaleza, su calidad y sus resultados pueden ser extendidos a otros contextos.

Las prácticas inclusivas deben estar caracterizadas por la instrumentación de estrategias didácticas que comprendan acciones sistémicamente coherentes, que se encuentren planificadas en función de los preceptos teóricos y los principios de la educación inclusiva, así como por su materialización desde formas que permitan la organización del proceso de enseñanza-aprendizaje para promover el máximo desarrollo posible en todo el alumnado. Dichas acciones tienen que estar orientadas a garantizar la estrecha relación entre todos los componentes del proceso bajo la rectoría de un fin, un propósito, un objetivo que se encuentre adecuadamente determinado en función de limitar las barreras en el aprendizaje y la participación de quienes se educan.

El hecho de considerar las prácticas inclusivas como la dimensión que decide el proceso de inclusión, también revela el valor del docente como agente capaz de incidir directamente en la identificación y eliminación de las barreras personales e institucionales, las que influyen en la disminución de oportunidades para que el alumnado con necesidades educativas especiales participe en las actividades socioeducativas y en su propio aprendizaje. El diseño curricular, su desarrollo y su evaluación deben estar concebidos de forma tal que promuevan prácticas inclusivas a partir de materializar las respuestas educativas de calidad que se precisen para cada caso.

El dilema de brindar una respuesta educativa individualizada en el contexto diverso de un salón de clases se resuelve por la vía didáctica y curricular, en la mayoría de los casos, siempre que los docentes estén en capacidad de diseñar currículos flexibles dirigidos con la ayuda de metodologías que han demostrado su efectividad, tanto en las prácticas de la Educación Especial tradicional, como desde las formas de aprendizaje basadas en grupos y de forma colaborativa. Encontrar una respuesta didáctica equilibrada y ajustada para promover un aprendizaje de calidad en los alumnos que se educan en condiciones de inclusión es un proceso complejo que se alcanza cuando se logren implementar acciones didáctico- metodológicas para compensar el desfasaje de las competencias curricular, académica y funcional del escolar con respecto al resto del grupo.

La Pedagogía Especial reconoce desde sus prácticas de enseñanza que las respuestas didácticas nunca deben ser rígidamente homogéneas, pues ante personas, necesidades y realidades diversas se impone la estructuración de estrategias personalizadas que garanticen una equiparación de oportunidades, una participación activa en el acto educativo, y mejorías significativas en los niveles de desarrollo integral de la personalidad de todo el alumnado. Sin embargo, tampoco debe fomentarse la marcada diferenciación de las tareas docentes, que al final, llegan a convertirse en un motivo y una justificación para la segregación y la exclusión.

\section{Metodología del estudio}

La estructuración sistémica de las acciones didácticas para lograr buenas prácticas inclusivas surge a partir de un proceso investigativo en el que los autores decidieron utilizar un tipo de investigación de alcance explicativo, teniendo en cuenta los criterios que ofrece Hernández (2014), al declarar que:

Los estudios explicativos van más allá de la descripción de conceptos o fenómenos o del establecimiento de relaciones entre conceptos; es decir, están dirigidos a responder por las causas de los eventos y fenómenos físicos o sociales. Como su nombre lo indica, su interés se centra en explicar por qué ocurre un 
fenómeno y en qué condiciones se manifiesta o por qué se relacionan dos o más variables. (p.128)

Las razones que justificaron este proceder se hallan en la novedad y pertinencia de la problemática que se investiga; lo que implicó, adentrarse en el estudio de una temática con resultados científicos dispersos, con diversidad de enfoques desde lo teórico y lo metodológico, y en muchas ocasiones, fuera del alcance de los docentes.

El procedimiento algorítmico empleado para la estructuración sistémica de las acciones didácticas que se ofrece se inició por la realización de un análisis crítico de la literatura especializada disponible, tanto de producción nacional como internacional. Este estudio permitió conocer las principales regularidades del estado del arte en cuestión, definiendo los preceptos y principios que en la actualidad son aceptados para gestionar el proceso de inclusión educativa de las personas con necesidades educativas especiales. Luego se construyó una primera versión del sistema de acciones didácticas, las que posteriormente fueron validadas por medio del criterio de expertos; para finalmente conformar la versión definitiva.

La decisión de utilizar el método de criterios de expertos se realizó tomando en consideración la opinión de Crespo (2009), el cual plantea que su uso es aconsejable en investigaciones con un enfoque teórico, con el propósito de evaluar la calidad y efectividad de la propuesta y comprobar la validez de las acciones y operaciones que se aplicarán.

Se entiende por experto a un individuo, grupo de personas u organizaciones capaces de ofrecer con un máximo de competencia, valoraciones conclusivas sobre un determinado problema, hacer pronósticos reales y objetivos sobre efecto, aplicabilidad, viabilidad y relevancia que pueda tener en la práctica la solución que se propone, así como brindar recomendaciones de qué hacer para perfeccionarla (Crespo, 2009).

De esta forma se puede asumir que la denominación del experto está íntimamente ligada a su competencia. Según Forgas (2003), la competencia profesional es el resultado de la integración esencial y generalizada de un complejo conjunto de elementos sustentados a partir de conocimientos, habilidades y valores, que se manifiestan a través de un desempeño profesional eficiente en la solución de los problemas de su profesión.

La forma más utilizada y confiable para completar la determinación de la competencia de los expertos, es mediante la determinación del coeficiente de competencia (coeficiente K) a partir de la autoevaluación de los posibles expertos. La experiencia demuestra que las personas con una elevada autoevaluación se equivocan menos que otras en sus predicciones (Crespo, 2009). Para la determinación del coeficiente K se empleó la metodología aprobada por el comité estatal para la Ciencia y la Técnica de Rusia en febrero de 1971, la cual toma como punto de partida la opinión del candidato sobre su nivel de conocimiento acerca del problema que se está resolviendo, basándose en las fuentes de argumentación que define el investigador con el propósito de determinar la competencia del experto.

Como fuentes de argumentación para este fin, en la presente investigación se propusieron las siguientes: 
Tabla 1.

Criterios para determinar los expertos.

\begin{tabular}{llll}
\hline FUENTES DE ARGUMENTACIÓN & ALTO & MEDIO & BAJO \\
\hline $\begin{array}{l}\text { Experiencia profesional que posee en la inclusión educativa del } \\
\text { alumnado con NEE. }\end{array}$ & 0,2 & 0,1 \\
\hline $\begin{array}{l}\text { Análisis de literatura especializada en el tema para el que se le } \\
\text { consulta. }\end{array}$ & 0,3 & 0,2 & 0,1 \\
\hline $\begin{array}{l}\text { Conocimientos sobre los fundamentos que sustentan la inclusión } \\
\text { educativa del alumnado con NEE. }\end{array}$ & 0,1 & 0,1 \\
\hline $\begin{array}{l}\text { Investigaciones o experiencias pedagógicas relacionadas con el } \\
\text { tema, dirigidas, tutoradas o consultadas. }\end{array}$ & 0,1 & 0,05 \\
\hline $\begin{array}{l}\text { Información que posee sobre el estado del arte en la temática } \\
\text { para la que se le consulta. }\end{array}$ & 0,05 & 0,05 & 0,05 \\
\hline $\begin{array}{l}\text { Intuición que expresa sobre la problemática para la que se le } \\
\text { consulta. }\end{array}$ & 0,05 & 0,05 & 0,05 \\
\hline $\begin{array}{l}\text { SUMA } \\
\text { SUla }\end{array}$ & 0,7 & 0,45 \\
\hline
\end{tabular}

En el proceso de selección de los expertos se determinó un grupo de candidatos conformado por 50 profesionales, con una labor afín al tema que se investiga y con experiencia en su desempeño. Estos autoevaluaron su nivel de competencia a partir de responder un cuestionario diseñado al efecto. Luego de aplicado dicho cuestionario, se calculó el coeficiente $\mathrm{K}$ empleando para ello la expresión $\mathrm{K}=1 / 2(\mathrm{kc}+\mathrm{ka})$, y se seleccionaron un grupo de 20 expertos cuyo coeficiente $\mathrm{K}$ oscilaba entre 0,8 y 1 , indicando un alto nivel de competencia en el tema que se investiga. El número seleccionado de expertos resulta adecuado según los criterios de Dalkay (citado por Zatsiorski, 1989) quien plantea que el número óptimo de expertos a seleccionar para una investigación debe de estar entre 15 y 30.

De los expertos seleccionados 15 poseen grado científico de Doctor en Ciencias Pedagógicas y cinco títulos académicos de Máster, 17 poseen además categorías docentes principales de profesor auxiliar y titular. Todos se desempeñan en educación, especial o superior, y tienen más de 20 años de experiencia profesional. Algunos poseen especializaciones en Oligofrenopedagogía, Psicopedagogía, Fisiología del desarrollo, Cultura Física, Educación Preescolar y Primaria e Integración escolar. La profesionalidad y diversidad de este grupo de expertos permite confiar en la fiabilidad y calidad de los criterios que se obtengan.

\section{Análisis y discusión de resultados}

Como vía para obtener criterios valorativos de los expertos acerca del sistema de acciones que se propone, se realizó una primera presentación y se aplicó una variante del Método de Rohrbach (Crespo, 2009).

Los expertos reunidos en una sesión de trabajo, se dividieron en cuatro grupos (de cinco personas) que escribieron en una hoja de papel tres ideas fundamentales en 10 minutos, al cabo 
de ese tiempo, entregaron sus ideas a uno de los tres grupos restantes y a su vez recibieron las de otro. A partir de ese momento la tarea de cada grupo consistió en desarrollar por escrito y en la propia hoja las ideas recibidas, pasándolas a otro grupo que a su vez desarrolló también las recibidas y las pasó a otro diferente. Éste proceso terminó cuando la hoja original de un grupo recorrió los tres grupos restantes, o sea, las tres ideas originales de cada grupo fueron desarrolladas tres veces. Durante el proceso, dentro de cada grupo las ideas se aclararon y se argumentaron, pero se prohibió criticarlas.

Una vez terminada esta sesión se contó con un grupo de ideas fundamentales, adecuadamente desarrolladas y fundamentadas, que expresaron el consenso de los expertos acerca de los aspectos esenciales del sistema de acciones didácticas que se propone. Estas ideas fueron: es pertinente la temática que se aborda, sobre todo en lo referido a la posibilidad de lograr buenas prácticas inclusivas; el perfeccionamiento de la estructuración didáctica del proceso de enseñanza-aprendizaje en contextos de inclusión favorecerá la atención a la diversidad de todo el alumnado; resulta muy importante continuar ampliando la fundamentación pedagógica de las acciones didácticas, de manera que se haga más evidente su finalidad educativa y su utilidad para la labor del maestro; se debe establecer una relación coherente entre el diagnóstico integral que se realiza en la escuela y el enfoque que debe tener el diagnóstico psicopedagógico para el logro de buenas prácticas inclusivas; es necesario ofrecer recomendaciones y sugerencias que permitan instrumentar las acciones didácticas para el logro de buenas prácticas inclusivas en las estrategias didácticas que se planean y se instrumentan en estos contextos educativos; y por último se sugirió lograr un adecuado balance entre los elementos teóricos y los metodológicos.

Una vez realizada esta primera consulta a los expertos y el correspondiente perfeccionamiento de las acciones didácticas-metodológicas sobre la base de sus criterios, se procedió a aplicar el método de preferencias. Según lo planteado por Crespo (2009) este es el método más empleado, por su exactitud, objetividad y rapidez, permite superar las limitaciones relacionadas con la complejidad de su aplicación y del procesamiento de los datos y alcanzar una imagen integral y más amplia del resultado científico sometido a valoración, reflejando las evaluaciones individuales de los expertos, las cuales podrán estar fundamentadas, tanto en un análisis estrictamente lógico como en su experiencia intuitiva.

Para aplicar el método se presentó a los expertos un segundo cuestionario. En este se solicitó que emitieran valoraciones acerca de criterios científicos formales (actualidad, novedad, relevancia y calidad), criterios sobre las posibilidades de perfeccionamiento de las prácticas inclusivas (valor pedagógico y posibilidades de aplicación) y criterios específicos sobre el sistema de acciones didácticas-metodológicas.

Los expertos debieron valorar los aspectos referidos, según la escala Muy adecuado (cinco), Bastante adecuado (cuatro), Adecuado (tres), Poco adecuado (dos), No adecuado (uno) y No sé (cero), elaborada de forma que exprese un orden decreciente de calidad. Un resumen de estas valoraciones permite afirmar que (once) aspectos fueron valorados como Muy adecuados y nueve como Bastante adecuados.

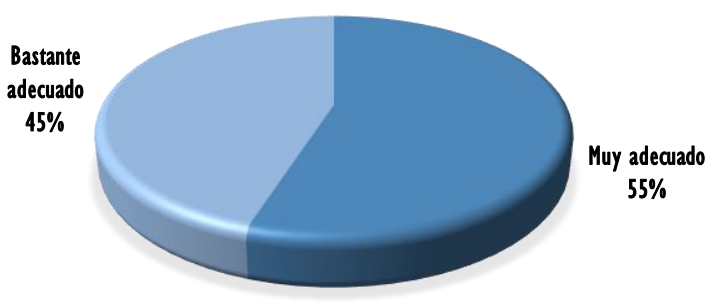


Al analizar la valoración general que realiza cada experto de las acciones didácticasmetodológicas se puede significar que doce lo consideraron Muy adecuado, siete Bastante adecuado y uno Adecuado. A pesar de que de forma general las valoraciones cuantitativas de los expertos fueron favorables se tomaron en cuenta sus recomendaciones para su perfeccionamiento.

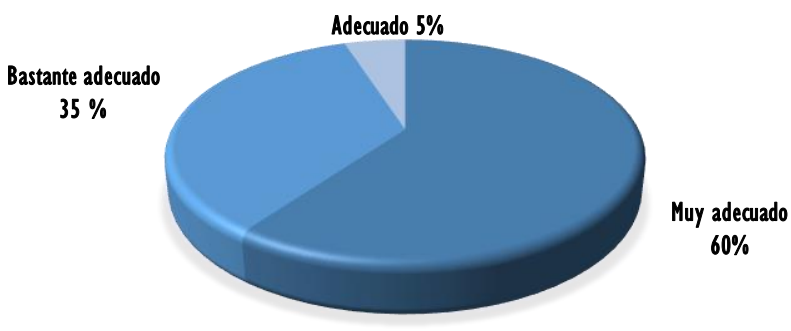

Una síntesis del sistema de acciones didácticas-metodológicas para el logro de buenas prácticas inclusivas, en su versión final, resultante de este proceso de perfeccionamiento a partir de la aplicación del criterio de los expertos en dos rondas de consultas, es presentado a continuación:

\section{Acciones para garantizar la interacción con los componentes personales del proceso de enseñanza-aprendizaje.}

En muchas ocasiones el proceso de inclusión educativa encuentra fuertes barreras, y estas no están relacionadas, precisamente, con la inexistencia de políticas inclusivas bien concebidas y sustentadas, sino por la forma en que las personas encargadas de materializarlas obstruyen o complejizan el desenvolvimiento de este proceso. En tal sentido, se reconoce por los autores del presente estudio la necesidad de encontrar "coherencia" en el accionar de todos los agentes educativos que influyen en la instrumentación de las estrategias de enseñanza, lo que significa, diseñar, instrumentar, evaluar y restructurar las acciones que se acometan de forma consensuada y bajo un enfoque sistémico; donde cada uno de los actores involucrados en el acto educativo debe jugar un rol protagónico y determinante. Estos actores, cuando se imbrican activamente en el desarrollo de un proceso de enseñanza-aprendizaje caracterizado por reconocer, respetar, atender y potenciar la diversidad humana se hallan en condiciones reales de estimular el máximo desarrollo posible de todo el alumnado.

Acción: Valoración de las potencialidades de los componentes personales del proceso de enseñanza-aprendizaje.

Esta acción se diseña con el propósito de determinar las potencialidades presentes en los agentes educativos convertidos en componentes personales del proceso de enseñanza-aprendizaje que se desarrolla en condiciones de inclusión educativa. Para instrumentar esta acción será necesario ejecutar operaciones tales como: determinar las características, las particularidades y las potencialidades que se hallan en cada uno de los agentes educativos encargados del proceso de inclusión educativa; evaluar las posibilidades de cada agente educativo para acometer las funciones que de él se demandan; así como promover desde el proceso de diagnóstico psicopedagógico los presupuestos y principios asumidos para la inclusión educativa.

Acción: Estimulación del actuar coherente de los principales actores que participan en la inclusión educativa desde el proceso de enseñanza-aprendizaje.

Esta acción se orienta a la actuación coherente de todos los actores que participan en el proceso de inclusión educativa. Para instrumentar esta acción será necesario ejecutar operaciones tales como: determinar los conocimientos, las destrezas y las actitudes a alcanzar por cada uno de los actores que constituyen componentes personales del proceso de enseñanza-aprendizaje; influir en los modos de actuación de los principales actores que participan del proceso de inclusión 
educativa a través de la ejecución de estrategias particulares que tomen en cuenta los conocimientos, las destrezas y las actitudes requeridas para lograr la inclusión educativa; promover el desarrollo de políticas inclusivas desde el accionar de los actores fundamentales involucrados en la atención a escolares con NEE desde contextos de inclusión educativa; fomentar el desarrollo de una cultura inclusiva desde el accionar de los actores fundamentales involucrados en la atención a escolares con NEE desde contextos de inclusión educativa; garantizar las condiciones básicas que se requieren para instrumentar prácticas inclusivas; así como la estimulación del desarrollo coherente del proceso de enseñanza-aprendizaje en condiciones de inclusión educativa a partir del involucramiento protagónico de sus principales actores.

Acción: Interrelación docente - escolar.

Esta acción se encuentra encaminada a lograr una interacción adecuada entre los docentes y los alumnos que se encuentran inmersos en el proceso de inclusión educativa. Para instrumentar esta acción será necesario ejecutar operaciones tales como: estructurar tareas docentes que faciliten la individualización de la enseñanza; promover la estimulación del desarrollo de cada escolar sobre la base del trabajo grupal y colaborativo; ejecutar actividades que promuevan la participación protagónica de todo el alumnado; diseñar un currículo flexible que se encuentre ajustado a las características y potencialidades individuales y grupales de todo el alumnado; lograr un adecuado clima pedagógico que facilite la eliminación de barreras en el aprendizaje y la participación; así como fomentar la ejecución de metodologías que promuevan la participación y el trabajo colaborativo desde la realización de tareas individuales (Enseñanza por proyectos, sociedades científicas e investigativas, círculos de interés, técnicas participativas de dinámicas grupales y de trabajo cooperativo).

\section{Acciones para la determinación, formulación, orientación y control de los objetivos.}

En la determinación, formulación, orientación y control del objetivo se pueden implementar acciones didácticas- metodológicas que contribuyan con la eliminación de las desigualdades, facilitando el acceso, la permanencia y el progreso de aquellos que más lo necesiten, favoreciendo así la inclusión socioeducativa.

Acción: Determinación y formulación del objetivo.

Esta acción se encamina a orientar a los docentes en la determinación y formulación de los objetivos a través de acciones didácticas-metodológicas para la planificación del proceso de enseñanza - aprendizaje de escolares con necesidades educativas especiales que se educan en contextos de inclusión. Para instrumentar esta acción será necesario ejecutar operaciones tales como: valorar en la determinación y formulación de los objetivos la activación de los procesos psíquicos cognoscitivos como la percepción, memoria, pensamiento, imaginación y las operaciones lógicas del pensamiento que promueva la igualdad de oportunidades para el aprendizaje; considerar en la determinación y formulación de los objetivos tanto los intereses del niño como los del grupo, los gustos y las motivaciones para el acceso a la inserción social; determinar cómo ha de proceder el alumno, qué materiales e instrumentos ha de utilizar y qué acciones debe desarrollar que contribuya a la regulación de la vida social y a la autorregulación de la actividad; considerar el carácter de sistema de los elementos que integran la estructura del objetivo; organizar las situaciones de aprendizaje de manera que se establezca la derivación gradual entre las habilidades que constituyen condiciones previas y las nuevas a lograr, a partir del diagnóstico individual y grupal; así como considerar desde la determinación y formulación de los objetivos la concepción de un currículo sustentado en el diseño universal de aprendizaje, valorando el carácter excepcional del diseño e instrumentación de las adaptaciones curriculares y los proyectos de grupo.

Acción: Orientación del objetivo.

Esta acción está dirigida a preparar a los docentes sobre el rol orientador del objetivo. Para instrumentar esta acción será necesario ejecutar operaciones tales como: precisar qué código de 
comunicación debe emplearse que responda a las necesidades educativas especiales es decir símbolos, signos, gestos; facilitar la comprensión y asimilación consciente de todo el alumnado de qué va hacer, reflexionar cómo lo va hacer, para qué lo va hacer; regular el volumen de la información que se brinda, ofrecer indicadores que precisen cómo llegar al fin, lo que facilitará la comprensión de significados; considerar desde el acto orientador de la actividad el establecimiento de relaciones significativas en el vínculo de la práctica con el material docente; condicionar estados emocionales positivos para ayudar a superar los sentimientos de minusvalía, baja autoestima y extrema dependencia; desarrollar la motivación, fundamentalmente la intrínseca; así como asegurar la concentración de la atención, para lo cual es determinante lograr un clima de comunicación asertivo, pausado y personalizado.

Acción: Valoración sobre la función de control de los objetivos.

Esta acción se encuentra orientada a preparar a los docentes sobre el papel del control del objetivo. Para instrumentar esta acción será necesario ejecutar operaciones tales como: observar en las diferentes situaciones evaluativas la reacción de los educandos con necesidades educativas especiales: vivencias afectivas, capacidad de trabajo, ritmo de trabajo y disposición para lograr el objetivo de la tarea; propiciar la autoevaluación crítica y cooperativa del escolar para el análisis del cumplimiento del objetivo de la tarea planteada; regular el volumen de habilidades que se va a evaluar, precisando paso a paso los indicadores para ofrecer seguridad y confianza; contribuir al desarrollo de una autovaloración positiva, lo que permite que el escolar pueda tener éxitos en el camino hacia el vencimiento del objetivo, tome confianza en sí mismo, desarrolle el autodominio, expectativas optimistas y adquiera habilidades para la vida en sociedad; identificar rasgos singulares del comportamiento en situaciones evaluativas observando su interés, motivación y la perseverancia para vencer con éxito una tarea; utilizar recursos variados para la evaluación y autoevaluación de las habilidades, carteles, signos gráficos, impulsos verbales; priorizar la colaboración del profesor en la aclaración de dudas ,solución de problemas; comparar y verificar los resultados así como concientizar al escolar de sus fortalezas y necesidades.

\section{Acciones para la determinación del contenido de la enseñanza.}

El contenido es una parte importante de la sabiduría, cultura y conocimientos que deben poseer las personas y en especial los educandos durante el aprendizaje, con el fin de conseguir las metas y objetivos propuestos y ser empleados en la vida cotidiana para resolver diferentes conflictos personales y/o profesionales. Estos se agrupan como componentes del proceso educativo en sistemas de: conocimientos, habilidades, valores y rasgos de la actividad creadora.

Acción: Revisión de documentos normativos que rigen el grado y la asignatura para conocer particularidades del contenido a impartir.

Esta acción se encamina hacia el estudio de los documentos normativos establecidos por el Ministerio de Educación donde se expresan las características, propósitos y exigencias de los contenidos para cada una de las asignaturas definidas en el currículo escolar. Para instrumentar esta acción será necesario ejecutar operaciones tales como: localizar los Programas, Orientaciones Metodológicas y otros documentos sugeridos por las autoridades educativas en todos los niveles; realizar un análisis de contenido, que permita identificar los beneficios y posibilidades que se hallan en el contenido para estructurar estrategias didácticas inclusivas; identificar en los contenidos propuestos, aspectos relevantes que pueden representar interés, necesidad o motivación de forma individual o colectiva; así como determinar las potencialidades que se expresan en los contenidos para establecer formas de trabajo grupal y cooperativa, diferenciando para cada caso, las tareas individualizadas a partir de los niveles de desarrollo de los alumnos.

Acción: Determinación de la correlación existente entre los conocimientos, habilidades, valores y rasgos de la actividad creadora, con el contenido a impartir y el diagnóstico grupal.

Esta acción se orienta a la determinación de los conocimientos, habilidades, valores y rasgos de la actividad creadora que se relacionan con el contenido a impartir y el diagnóstico grupal. Para 
instrumentar esta acción será necesario ejecutar operaciones tales como: analizar las potencialidades, necesidades y motivaciones de los educandos; seleccionar los conocimientos, habilidades, valores y rasgos de la actividad creadora que pueden ser tratados desde las diferentes formas de organización de la enseñanza; valorar los conocimientos, habilidades, valores y rasgos de la actividad creadora que respondan a las potencialidades, necesidades y motivaciones detectadas anteriormente en los educandos; así como establecer los nexos entre cada uno de ellos.

Acción: Apropiación del contenido.

Esta acción se orienta hacia la instrumentación de estrategias para la apropiación del contenido a partir del análisis previo de las características generales y particulares de los educandos. Para instrumentar esta acción será necesario ejecutar operaciones tales como: confirmar el dominio de los conocimientos previos que poseen los educandos sobre el contenido a impartir; determinar la vía más idónea para introducir el contenido; garantizar los medios y recursos necesarios; interrelacionar los conocimientos previos que poseen los educandos, con las características del contenido a impartir; dosificar el contenido atendiendo a sus niveles de complejidad y las formas metodológicas establecidas para su tratamiento; determinar los elementos del contenido que se encuentran en correspondencia con los niveles de desarrollo de cada alumno; identificar las formas de organización de la enseñanza, así como la pertinencia de los métodos y procedimientos más eficaces para promover la apropiación del contenido definido en el currículo por todo el alumnado; ofrecer los niveles de ayuda y la estimulación que requieren los educandos según sus necesidades y potencialidades; generar nuevas situaciones o similares para que transfiera los conocimientos adquiridos; elaborar conclusiones del contenido impartido; así como la comprobación de los conocimientos adquiridos en los estudiantes, a partir de la demostración de habilidades, valores y rasgos creativos.

\section{Acciones para la selección y desarrollo de los métodos de la enseñanza.}

El fin de la inclusión educativa, entre muchos factores, estará determinado por el logro de las buenas prácticas que se materialicen en el contexto del proceso de enseñanza-aprendizaje. Allí deberán instrumentarse las mejores estrategias didácticas para promover el desarrollo del alumnado, las que en correspondencia con la complejidad y las particularidades de la atención a los escolares con necesidades educativas especiales en contextos inclusivos, ponderan la selección adecuada de los métodos y procedimientos a la hora de individualizar la enseñanza en el contexto de una educación inclusiva.

Seleccionar un buen método de enseñanza significa que este se encuentre en absoluta y total correspondencia con los objetivos que se propongan para cada actividad, sin embargo, cuando el método se selecciona tomando en cuenta que a través de él se atenderá un grupo diverso de escolares el proceso que se siga debe estar marcado además: por un profundo conocimiento del diagnóstico psicopedagógico, tanto individual, como grupal; por un dominio de las potencialidades del alumnado, de sus estilos de aprendizaje y sus preferencias sensoriales, de los tipos de inteligencia que predominan en ellos y de los procedimientos metodológicos para incentivarlas de forma más efectiva; por las particularidades del currículo y las vías para desarrollarlo bajo los principios de la inclusión educativa; así como por la posibilidad de emplear formas de agrupamiento flexibles en correspondencia con los factores antes expuestos.

Acción: Selección de métodos que favorezcan a la inclusión educativa.

Esta acción se orienta a la determinación de los mejores métodos que favorecen el proceso de inclusión educativa. Para instrumentar esta acción será necesario ejecutar operaciones tales como: analizar la pertinencia del método seleccionado hacia el logro del objetivo y la apropiación del contenido por "todo" el alumnado; valorar la pertinencia del desarrollo del método seleccionado en correspondencia con los medios y recursos didácticos existentes, así como por las condiciones temporoespaciales disponibles; valorar la pertinencia del método seleccionado en correspondencia con las características y particularidades tanto individual, como grupal del 
alumnado; garantizar que el método seleccionado no propicie modos de actuación tendientes a la segregación y a la exclusión ni en los alumnos ni en el colectivo pedagógico; determinar con precisión todos los procedimientos necesarios para estructurar un método de enseñanza que se ajuste y se corresponda con los principios y presupuestos de la inclusión educativa; evaluar el proceso de selección de los métodos a partir de la consulta con profesionales y especialistas competentes.

\section{Acciones para la selección, aplicación y evaluación de los recursos didácticos y medios de enseñanza.}

El uso de los recursos didácticos y los medios de enseñanza como mediadores en el proceso de enseñanza-aprendizaje en el proceso de inclusión educativa debe ser consciente e intencional para guiar las acciones y su secuenciación en función de alcanzar determinadas metas de aprendizaje; su implementación en la práctica educativa no puede ser automática, ni espontánea, sino controlada y planificada, requieren de la selección, proyección y control en su ejecución, además de la valoración de lo afectivo y lo motivacional para la satisfacción de las necesidades educativas especiales de los escolares.

Acción: Selección del recurso didáctico o del medio de enseñanza.

Esta acción se diseña con el propósito de determinar el recurso didáctico o el medio de enseñanza que utilizará durante el proceso de enseñanza aprendizaje. Para instrumentar esta acción será necesario ejecutar operaciones tales como: Profundizar en las características de los recursos didácticos y de los medios de enseñanza; estudiar la clasificación de los recursos didácticos y de los medios de enseñanza; seleccionar el recurso didáctico y de los medios de enseñanza teniendo en cuenta la diversidad que tiene en su grupo y la utilidad del mismo para alcanzar responsabilidad, solidaridad, disciplina para ser demostrados en sus modos de actuación y proyectar en qué momento los utilizará.

Acción: Ubicación de los escolares en situación de aprendizaje (Motivación).

Esta acción se diseña con el propósito de colocar a los escolares en situación de aprendizaje para la obtención de efectividad en la utilización del recurso didáctico o medio de enseñanza. Para instrumentar esta acción será necesario ejecutar operaciones tales como: Concretar la respuesta educativa en función del diagnóstico; potenciar el desarrollo y formación de habilidades sociales, comunicativas en los escolares; determinar el estilo de aprendizaje de sus escolares; organizar previamente para proyectar las interacciones entre los escolares. (Cómo desean trabajar); creación de condiciones físico- ambientales. (Silencioso, iluminado, activos, áreas abiertas, en el aula, fuera del aula, entre otros); contribuir, por su esencia comunicativa al enriquecimiento de las experiencias sensoriales de los alumnos y a maximizar la motivación por aprender.

Acción: Activación de los conocimientos. (Proceso de atención, interacciones, intereses, competencia curricular, modalidad de mediación).

Esta acción se diseña con el propósito de impulsar los conocimientos teniendo en cuenta la orientación propiamente cognitiva y de apoyo e inducción del proceso de enseñanza-aprendizaje hacia un nuevo conocimiento mediante el uso del recurso didáctico o medios de enseñanza. Para instrumentar esta acción será necesario ejecutar operaciones tales como: Ofrecer la posibilidad de una respuesta en concordancia con la estructura cognitiva de los escolares; determinar el nivel de atención. (Cuando quiere aprender); mantener la atención durante las actividades de aprendizaje; considerar el nivel de desarrollo de los escolares.; controlar la calidad, exactitud y velocidad del aprendizaje, así como la experiencia que tienen los escolares; prevenir los niveles de ayuda pedagógica que debe ofrecer (Alerta, Llamado de atención, Reformulación de la instrucción, Aprobación o crítica, Estimulación, Movilización de la actividad mental, Preguntas de apoyo, Precisiones de ideas, Organización del proceso lógico, Demostración); poner al alumno en posición de éxito, en condiciones favorables para desarrollar el aprendizaje; focalizar los factores potencialmente generadores de limitaciones en el desarrollo del proceso de enseñanza- 
aprendizaje; estructurar la estimulación psicopedagógica desde posiciones preventivas, así como el logro del enriquecimiento de la experiencia de los escolares: proporcionar que los escolares expresen espontáneamente sus conocimientos a partir del recurso utilizado; elevación de la autoestima de los escolares, en la interacción con múltiples agentes y agencias de socialización mediante la utilización de los mismos; propiciar la incorporación del escolar mediante el uso del recurso a la diversidad de las redes sociales, entendida como el desarrollo de la sociabilidad.

Acción: Evaluación del aprendizaje mediante el uso del recurso o medios de enseñanza.

Esta acción se diseña con el propósito de evaluar el nivel de aprendizaje de los escolares mediante la utilización del recurso o el medio y medir efectividad. Para instrumentar esta acción será necesario ejecutar operaciones tales como: Determinar la modalidad de evaluación que utilizará teniendo en cuenta las características del grupo. (Oral, escrita, y si les gusta ser evaluados); valorar la efectividad y satisfacción de los escolares en el uso del recurso o de los medios.

\section{Acciones didácticas - metodológicas para la evaluación del aprendizaje.}

Es prioritario y de máximo interés establecer un modelo de evaluación coherente con los principios de inclusión: participación y aprendizaje de todos. Una evaluación continua, flexible y dinámica, que acompañe todo el proceso de enseñanza aprendizaje y tenga como propósito fundamental proporcionar información -al inicio, durante y al final del proceso-, para la toma de decisiones. Es decir, que permita conocer el punto de partida de los estudiantes con relación con los aprendizajes esperados, retroalimentar y ajustar el proceso de enseñanza acorde a las características y necesidades de los estudiantes, y comprobar si se han logrado o no, y en qué medida, los aprendizajes.

Acción: Activación de los conocimientos. (Diagnóstico)

Esta acción se diseña con el propósito de impulsar los conocimientos teniendo en cuenta la orientación propiamente cognitiva y de apoyo e inducción del proceso de enseñanza-aprendizaje hacia los conocimientos que deben poseer del contenido objeto de evaluación. Para instrumentar esta acción será necesario ejecutar operaciones tales como: Observar la actitud, predisposición, participación activa y la autonomía personal que presentan los escolares para ser evaluados; determinar el grado de asimilación de los escolares atendiendo a la diversidad; determinar el ritmo y estilo de aprendizaje de los escolares para determinar el tipo de evaluación; explorar las capacidades que poseen los escolares para retener conocimientos que se adquieren; evaluar el nivel de conocimientos que poseen los escolares sobre los contenidos que se evaluarán; organizar previamente para proyectar las interacciones entre los escolares. (Cómo desean ser evaluados); crear las condiciones físico- ambientales (Silencioso, iluminado, activos, áreas abiertas, en el aula, fuera del aula, etc.); contribuir a maximizar la motivación por ser evaluados.

Acción: Determinación del objetivo que se evaluará.

Esta acción se diseña con el propósito de determinar el objetivo que se evaluará a partir del contenido seleccionado. Para instrumentar esta acción será necesario ejecutar operaciones tales como: Profundizar en los contenidos más importantes que requieren ser evaluados; determinar lo esencial del contenido que se evaluará; determinar la habilidad o habilidades a evaluarse, así como su estructura interna; determinar el grado de flexibilidad atendiendo a la diversidad; elaborar el objetivo de evaluación de manera clara, que especifique resultados, pero prestando atención también a los procesos; determinar el nivel de asimilación que se debe lograr en la evaluación; informar a los escolares los objetivos de la evaluación de modo que la evaluación se vea como un proceso positivo que destaca los progresos individuales.

Acción: Selección del tipo de evaluación.

Esta acción se diseña con el propósito de seleccionar el tipo de evaluación que utilizará. Para instrumentar esta acción será necesario ejecutar operaciones tales como: Profundizar en los tipos de evaluación; modelar anticipadamente el tipo de evaluación seleccionada para determinar su 
eficacia; determinar en qué momento realizará la evaluación; selección del tipo de evaluación que aplicará que responda al diagnóstico del grupo, al contenido y al propósito.

Acción: Selección de la actividad que se evaluará.

Esta acción se diseña con el propósito de determinar el tipo de actividad que será evaluada. Para instrumentar esta acción será necesario ejecutar operaciones tales como: Determinar la técnica o modalidad de evaluación que se aplicará (Pruebas, controles, preguntas orales y escritas, de forma prevista e imprevista. Tareas, trabajos y proyectos, individuales y en grupo; ejercicios de clase, deberes, observaciones, etc.); seleccionar un lenguaje claro asequible a la diversidad en la actividad seleccionada; expresar el objetivo de forma clara precisa en que el escolar comprenda lo que se le está evaluando.

Acción: Valorar la efectividad y satisfacción de los escolares en la evaluación.

Esta acción se diseña con el propósito de constatar la efectividad y grado de satisfacción de los escolares en la evaluación. Para instrumentar esta acción será necesario ejecutar operaciones tales como: Analizar conjuntamente los resultados y el porqué de esos resultados; escuchar opiniones de los escolares acerca de los resultados de la evaluación realizada. Así como el grado de satisfacción que reflejan; medir efectividad de la evaluación.

\section{Acciones para la estructuración de las formas de organización de la enseñanza.}

Alcanzar la calidad educativa en el tratamiento a la diversidad desde un planteamiento inclusivo, exige al maestro reconocer la clase como la forma de organización fundamental del proceso de enseñanza-aprendizaje, la misma ofrece la posibilidad de organizar de distintas maneras el trabajo con los alumnos, por ejemplo: la excursión, seminario, clases prácticas, trabajo en equipos, en huertos y parcelas, debate de una película o video, el panel, entre otras.

Acción: Estudio panorámico del currículo general e institucional correspondiente al grupo/grado.

Esta acción conlleva a realizar operaciones que permitirán durante la auto preparación del maestro familiarizarse con el currículo general e institucional con el que trabajará en el grupo/clase; identificar las posibilidades que brinda cada programa para actuar con flexibilidad, así como en las diferentes asignaturas los contenidos que presentan mayores dificultades para la asimilación por los estudiantes, sus posibles causas y las mayores dificultades en cuanto a cómo impartirlos.

Acción: Organización de la clase en equipos de trabajo.

Subyacen operaciones orientadas a la organización del grupo en pequeños subgrupos denominados equipos base, con una composición heterogénea en rendimiento y capacidad (5 a 6 integrantes). Distribuir los roles o cargos en cada equipo, indicando que las distintas tareas propias de cada rol sean rotativas, ya que todos deben ejercer todos los cargos.

Establecer de forma colaborativa con el grupo de educandos, las normas de funcionamiento para el trabajo en equipo, las que regularán el funcionamiento del grupo, por ejemplo: compartirlo todo, pedir la palabra antes de hablar, escucharse mutuamente, aceptar las decisiones de la mayoría, ayudar a los compañeros, pedir ayuda cuando se necesite, no rechazar la ayuda de un compañero, cumplir las tareas orientadas, participar en todos los trabajos y actividades del equipo, entre otras que se considere.

\section{Referencias}

Ainscow, M. (2004). El desarrollo de sistemas educativos inclusivos: ¿Cuáles son las palancas de cambio? Journal of Educational Change, 5(4), 1-20.

Blanco, G, R. (2006). La equidad y la inclusión social: uno de los desafíos de la educación. REICE - Revista Electrónica Iberoamericana sobre Calidad, Eficacia y Cambio en Educación, 4(3), 1-15. 
Booth, T., Ainscow, M., Black-Hawkins, K., Vaughan, M., \& Shaw, L. (2002). Centre for Studies on Inclusive Education (CSIE), Bristol UK 2000.

Booth, T. y Ainscow, M. (2002). Indice de inclusión. Desarrollando el aprendizaje y la participación en las escuelas. Santiago de Chile: ORLEAC/UNESCO.

Castel, R. (1995). La metamorfosis de la cuestión social. Buenos Aires: Paidós.

Crespo, T. (2009). Métodos de la Prospectiva en la Investigación pedagógica. La Habana: Educación Cubana.

Echeita, G., \& Ainscow, M. (2011). La educación inclusiva como derecho. Marco de referencia y pautas de acción para el desarrollo de una revolución pendiente. II Congreso Iberoamericano de Síndrome de Down.

Escribano, A., \& Martínez, A. (2013). Inclusión educativa y profesorado inclusivo: aprender juntos para aprender a vivir juntos (Vol. 122). Narcea Ediciones.

Forgas, J. (2003). Modelo para la formación profesional, en la Educación Técnica y profesional, sobre la base de Competencias Profesionales, en la rama Mecánica. (Tesis doctoral). Universidad de Santiago de Cuba: Santiago de Cuba.

González, R., Rodríguez, B., Colás, A., \& Ramírez, E. T. (2019). La inclusión educativa: retos y perspectivas desde la Agenda 2030. Pedagogía 2019. La Habana: Sello editor Educación Cubana.

Granada, M., Pomés, M. P., \& Sanhueza, S. (2013). Actitud de los profesores hacia la inclusión educativa.

Hernández, R., Fernández, C., \& Baptista, P. (2014). Metodología de la investigación. Sexta Edición. México: Industria Editorial Mexicana.

Pietroni, M. (2016). Significado de inclusión, calidad educativa y su relación en el nivel secundario: tensión en una política de la provincia de Santa Fe entre un programa, la voz de los docentes y posibles alternativas conceptuales. Santa Fé. Universidad Nacional del Litoral. Tesis en opción al título de Master en Políticas públicas para la educación.

Ramírez, C, S. (2010). Educación inclusiva y la estimulación temprana. ARJÉ Revista de Postgrado FACE-UC, 4(1),153-170.

Real Academia Española (2014). Diccionario de la lengua española (23. ${ }^{a}$ ed.) Recuperado de UNESCO (2008). Educación inclusiva: camino hacia el futuro. Unesco.

Zatsiorski, V. (1989). Metrología Deportiva. Moscú: Editorial Planeta. 